Eur. J. Clin. Chem. Clin. Biochem.

Vol. 29, 1991, pp. 51-55

(C) 1991 Walter de Gruyter \& Co.

Berlin · New York

\title{
Neuronal Acetylcholinesterase Levels in Cerebrospinal Fluid and Serum Determined by a Specific and Sensitive Immunoassay ${ }^{1}$ )
}

\author{
By M. Mäder ${ }^{1}, K$. Soerensen ${ }^{2}, T h$. Wiedmann $^{1}$, U. Dickmann ${ }^{1}$ and K. Felgenhauer ${ }^{1}$ \\ 1 Neurologische Klinik der Universität Göttingen \\ ${ }^{2}$ Institute of Molecular Biology and Biochemistry der Universität Bern ${ }^{2}$ )
}

(Received November 11, 1989//July 6/November 2, 1990)

Summary: Acetylcholinesterase levels were determined in cerebrospinal fluid and serum of 272 patients with various neurological disorders. The patients were ordered in 13 diagnostic groups. The assay employed was an antigen capture assay based on an immobilized monoclonal antibody selective for neuronal acetylcholinesterase (Rasmussen et al., Clin. Chim. Acta 166 (1987) 17-25 (1)).

In $100 \%$ of the cases the acetylcholinesterase levels in cerebrospinal fluid were $500-10000$ fold higher than expected for a regular serum-derived protein $(0.1-0.5 \%)$ at the intact blood-brain barrier. In both compartments the test values showed wide variations, which were much greater in serum than in cerebrospinal fluid. No specific relationship was discernible between the values obtained and any of the diagnostic groups, although decreased levels of acetylcholinesterase were found in bacterial and viral meningitis, and elevated levels were found in groups with more chronic diseases.

A possible transfer of acetylcholinesterase from cerebrospinal fluid to blood is discussed.

\section{Introduction}

The search for biochemical markers of brain destruction and disease in the circulating blood has been unsuccessful so far (2). The extracellular space of other organs, e.g. muscle and liver is open to the free water compartment of the body, whereas the bloodbrain barrier virtually blocks the direct transfer of proteins in both directions. The extracellular space of the central nervous system is thought to be cleared via the cerebrospinal fluid compartment (3), but it is conceivable that this "sink" function is restricted to

') Supported by the Deutsche Forschungsgemeinschaft, SFB 330.

2) Present address: K. Soerensen, Equichem Research Institute LTD, 393 Old Country Road, Carle Place, New York 11 514, UIS.A.

3) Enzymes:

Acetylcholinesterase (EC 3.1.1.7)

Butyrylcholinesterase (EC 3.1.1.8) superficial layers adjacent to the free cerebrospinal fluid space, as evidenced by the clearance of the carcinoembryonic antigen from deep parenchymal metastases (4).

In order to serve as process markers in the blood, proteins ought to be of high molecular mass, soluble in aqueous phases and slowly catabolized in the body. In all these aspects acetylcholinesterase ${ }^{3}$ ) represents a promising candidate, the more since Chubb and coworkers found that the acetylcholinesterase of the cerebrospinal fluid is derived from the central nervous system (5).

Histochemical studies show acetylcholinesterase widely distributed in the brain, where it fills predominantly the extracellular space of several brain nuclei, e. g. nucleus caudatus, lentiformis, amygdalae, accumbens, ventriculus anterior thalami and geniculatus lateralis (6). 
Acetylcholinesterase appears in the amniotic fluid of fetuses with neural tube defects, obviously having leaked from the early cerebrospinal fluid space (7). Acetylcholinesterase, when measured, has to be differentiated from the erythrocyte isoenzyme. As Rasmussen and coworkers have obtained a monoclonal antibody largely selective for the neuronal isoenzyme (1), we were able to investigate the distribution of the neuronal acetylcholinesterase in cerebrospinal fluid and serum of a large number of patients.

\section{Materials and Methods}

\section{Samples}

Cerebrospinal fluid and blood samples were taken by lumbarand venipuncture respectively, from 272 patients with various neurological disorders (151 males, 121 females; age range 17 to 78 years). For reference purposes, blood samples were drawn from 9 healthy persons (students) aged about 25. The blood samples were centrifuged after clotting for one hour. Blood and cerebrospinal fluid samples were then frozen in aliquots at $-70^{\circ} \mathrm{C}$.

\section{Determination of acetylcholinesterase}

The immunoassay for determination of acetylcholinesterase was exactly as described by Rasmussen et al. (1), who also reported the accuracy, specificity and sensitivity of the assay. The monoclonal antibody 4F19 was employed in this study. Values presented are the average of three repeats.

\section{Assay of blood-brain barrier function}

Blood-brain barrier function was assayed according to 1.c. (8) and (2) by determination of the albumin quotients (cerebrospinal fluid: serum); an albumin quotient $\left(\mathrm{Q} \times 10^{3}\right) \geq 5$ indicates blood-brain barrier impairment.

\section{Results}

Figure 1 shows the distribution of the test values of cerebrospinal fluid and serum. Cerebrospinal fluid values show a standard distribution, whereas the distribution of the serum values is skewed towards the lower values, including a considerable number of measurements below the detection limit. Matrix and methodical effects were ruled out by mixing experiments (data not shown); see also 1.c. (1). The relationship between the increasing serum values and the increasing cerebrospinal fluid values is shown in figure 2.

In figure 3 , the 544 test values are presented in a Boxand-Whisker Plot, which allows direct comparison of values with skewed distribution. The patients are ordered in 13 diagnostic groups and a reference group for serum values. There is a wide variation of cerebrospinal fluid and serum values in all groups. The variation is lowest, however, in the reference group

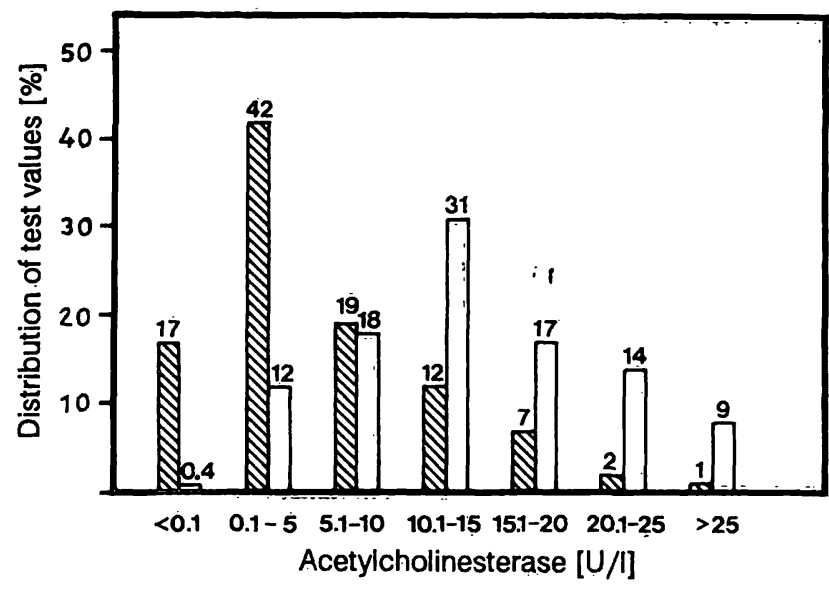

Fig. 1. Distribution of acetylcholinesterase values of serum and cerebrospinal fluid in arbitrary measurement classes ranging from $0.1->25 \mathrm{U} / \mathrm{l}$. $(1 \mathrm{U}=1 \mu \mathrm{mol}$ of substrate hydrolysed per minute at $25^{\circ} \mathrm{C}$ ). Hatched bars, serum; white bars, cerebrospinal fluid; $<0.1$, values below the detection limit.

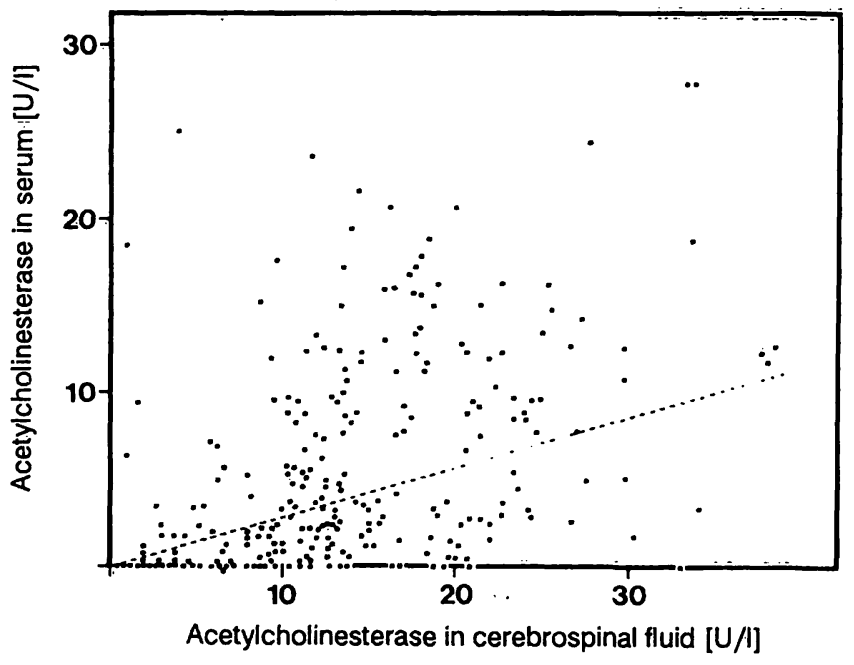

Fig. 2. Acetylcholinesterase values of the sera of 272 patients were plotted versus the corresponding values of the cerebrospinal fluids. The dashed line represents $y=a x$ where a $(1: 4)$ is the quotient: $\operatorname{median}_{(\text {serum) }} / \mathrm{me-}$ $\operatorname{dian}_{\text {(cerebrospinal nuid) }}$ of the average test value (see fig. 3 ). $1 \mathrm{U}=1 \mu \mathrm{mol}$ of substrate hydrolysed per minute at $25^{\circ} \mathrm{C}$.

of healthy persons which were all of similar age. With the exception of groups 5 and 8 (polyneuropathy and . transitoric ischaemic attack, respectively), the various groups show serum values below the detection limit, although this is less pronounced in groups 4 and 11 (inflammatory disease and miscellaneous diseases respectively); compare table 1.

In all groups the median of cerebrospinal fluid values is above the median of serum values.

Serum values showing the greatest deviation from the average value fall into two categories: those below the detection limit (17\%), and those which are as high 


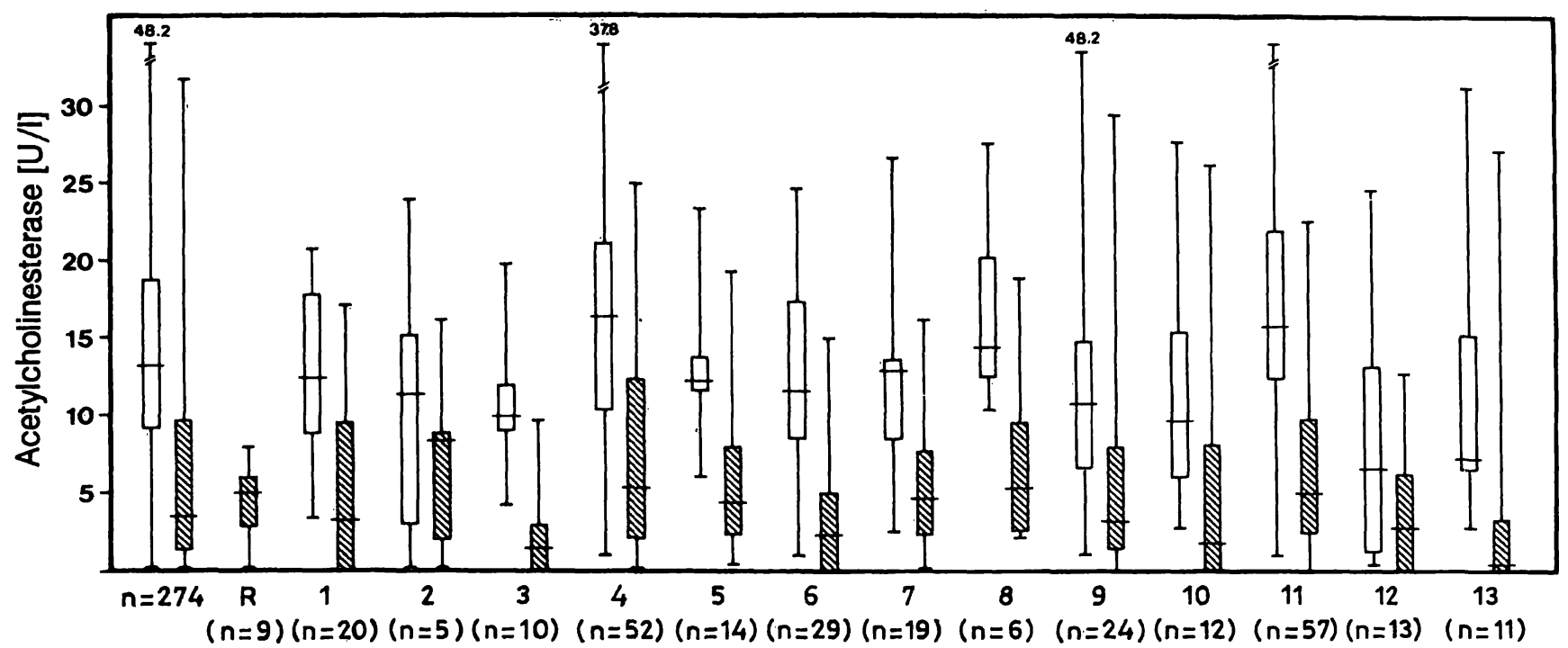

Fig. 3. Box- and Whisker-Plot of acetylcholinesterase measurements in serum and cerebrospinal fluid of 272 patients ordered in 13 diagnostic groups. Additionally there is one reference group of serum (healthy students) and one group of all values measured in serum and cerebrospinal fluid. 272: all values of 272 patients, R: reference group serum, 1: epilepsy, 2: brain haemorrhage, 3: tumours, 3: inflammatory diseases, 5: polyneuropathy, 6: disk protrusion, 7: multiple sclerosis, 8: transitoric ischaemic attack, 9: brain infarction, 10: psychosis, 11: miscellaneous diseases, 12: bacterial meningitis, 13: viral meningitis. The line crossing the rectangles represents the median or $50 \%$ of the measured values. The lower and upper limits of the rectangles represent 25 or $75 \%$ of the values, respectively. The rectangle itself again represents $50 \%$ of the measured values.

Hatched rectangles, serum; white rectangles, cerebrospinal fluid. $1 \mathrm{U}=1 \mu \mathrm{mol}$ of substrate hydrolysed per minute at $25^{\circ} \mathrm{C}$.

Tab. 1. Distribution of brain-barrier impairments, values below the detection limit in the serum, and serum values as high or higher than the corresponding cerebrospinal fluid values among the diagnostic classes; $n$, number of patients studied; $C$, number of cases; \%, percentage of cases.

\begin{tabular}{|c|c|c|c|c|c|c|c|}
\hline \multirow[t]{2}{*}{ Diagnostic classes } & \multirow[t]{2}{*}{$\mathrm{n}$} & \multicolumn{2}{|c|}{$\begin{array}{l}\text { Brain-barrier } \\
\text { impairment }\end{array}$} & \multicolumn{2}{|c|}{$\begin{array}{l}\text { Zero values } \\
\text { in serum }\end{array}$} & \multicolumn{2}{|c|}{$\begin{array}{l}\text { Serum values } \\
\geq \text { CSF value }\end{array}$} \\
\hline & & $\mathrm{C}$ & $\%$ & $\mathrm{C}$ & $\%$ & $\mathrm{C}$ & $\%$ \\
\hline Epilepsy & 20 & 6 & 30 & 5 & 25 & 6 & 30 \\
\hline Brain haemorrhage & 5 & 2 & 40 & 1 & 20 & 3 & 60 \\
\hline Tumours & 10 & 5 & 50 & 3 & 30 & 1 & 10 \\
\hline Inflammatory diseases & 52 & 23 & 44 & 3 & 6 & 10 & 19 \\
\hline Polyneuropathy & 14 & 7 & 50 & 0 & 0 & 3 & 21 \\
\hline Disk protrusion & 29 & 18 & 62 & 9. & 31 & 6 & 21 \\
\hline Multiple sclerosis & 19 & 9 & 47 & 5 & 26 & 5 & 26 \\
\hline Transitoric ischaemic attack & 6 & 5 & 83 & 0 & 0 & 1 & 16 \\
\hline Brain infarction & 24 & 16 & 67 & 5 & 21 & 4 & 17 \\
\hline Psychosis & 12 & 4 & 33 & 5 & 42 & 1 & 8 \\
\hline Miscellaneous diseases & 57 & 19 & 33 & 2 & 4 & 10 & 18 \\
\hline Bacterial meningitis & 13 & 9 & 69 & 5 & 38 & 1 & 8 \\
\hline Viral meningitis & 11 & 5 & 45 & 5 & 45 & 3 & 27 \\
\hline
\end{tabular}

or even higher in serum than in cerebrospinal fluid of the same person $(20 \%)$. Most of these latter values are in the high range in both compartments $(>10 \mathrm{U}$, fig. 1). In table 1 , the percentage of blood-brain barrier impairments per group is compared with the percentage of values below the detection limit and with the percentage of the values which are higher or as high in serum as in cerebrospinal fluid. The data from figure 3 is supplemented in various ways. Thus, groups 12 and 13 (bacterial and viral meningitis re- spectively, which are undoubtedly serious diseases with decreased acetylcholinesterase levels in cerebrospinal fluid and serum) (fig. 3) show increased numbers of blood-brain barrier impairments and an increased number of values below the detection limit in the sera (tab. 1). Since, in addition to groups 12 and 13 , high percentages of blood-brain barrier impairments are also found in groups 6,8 , and 9 (disk protrusion, transitoric ischaemic attack, and brain infarction), there is no correlation between blood- 
brain barrier impairment and the values below the detection limit. Serum values, which are as high or higher than the corresponding values of cerebrospinal fluid, are more or less equally distributed throughout the diagnostic groups, with the exception of group 2 (brain haemorrhage). Group 2, however, is one of the two groups ( 2 and 8 ) with the smallest number of cases.

Finally, groups 4 and 11 (inflammatory and miscellaneous diseases), which are the largest groups (52 and 57 cases, respectively), both show a very low percentage of values below the detection limit (tab. 1). They show, however, widely varying elevated values of acetylcholinesterase in their cerebrospinal fluid, and they include the highest values of all measured in this study (fig. 3). These groups are the most heterogeneous and they represent the more chronic diseases. These two groups show average values of all the other parameters studied (tab. 1).

\section{Discussion}

It has not been possible in the past to determine reliably the amount of acetylcholinesterase present in biological fluids, since there is neither a substrate nor an inhibitor absolutely specific for acetylcholinesterase or butyrylcholinesterase ${ }^{3}$ ) (9). Erroneous acetylcholinesterase levels have been determined mainly in the serum, which contains an overwhelming preponderance of butyrylcholinesterase. In the present study, an immunoassay was employed based on a monoclonal antibody selective for neuronal acetylcholinesterase (1). There was a slight cross-reactivity $(=10 \%)$ with erythrocyte acetylcholinesterase (1). Blood-contaminated cerebrospinal fluid samples, however, did not show elevated acetylcholinesterase levels, thus proving the reliability of the assay.

Eighty percent of the acetylcholinesterase levels determined in the cerebrospinal fluid of 272 patients with various neuropsychiatric disorders were higher (1.5-10 fold) than the corresponding levels in the sera. In addition to these results, the finding that increasing serum values correspond to increasing cerebrospinal fluid values (fig. 2) supports the idea of a brain to blood transfer of acetylcholinesterase. Further evidence lends support to the above idea; thus there were many values below the detection limit $(17 \%)$ in the patients' sera, while the corresponding cerebrospinal fluid values were well above the detection limit and lay over a wide range. Finally, it is well established that about $90 \%$ of the regular cerebrospinal fluid protein originates from the serum (10). It is therefore relevant to ask whether at least some part of acetylcholinesterase in the cerebrospinal fluid may originate from the serum. Neuronal acetylcholinesterase has a relative molecular mass of 290000 , corresponding to a hydrodynamic radius of $5.8 \mathrm{~nm}(58 \AA)$ (10). At the intact blood-cerebrospinal fluid barrier the cerebrospinal fluid level would thus reach not more than $0.1 \%$ of the serum level $(100 \%)$. The average cerebrospinal fluid level determined in this study is, however, 4 fold $(400 \%)$ that of the corresponding serum level. Even in those cases $(10 \%)$ where the serum levels are above the cerebrospinal fluid levels the latter are at least $50 \%$ of the serum concentration. This by far exceeds the $0.1 \%$ cerebrospinal fluid concentration expected for a regular serum protein of the size of acetylcholinesterase. Therefore it is justified to assume that the total amount of acetylcholinesterase in the cerebrospinal fluid originates from the nervous parenchyma of the brain. Compared with the central nervous system, the mass of cholinergic neurons in the periphery is very small indeed. Acetylcholinesterase is the second neuronal enzyme to be described exhibiting cerebrospinal fluid/serum rations $\geq 1$ (the first was neuron-specific enolase (11)), suggesting that it originates in the brain. Detection of brain-derived proteins in cerebrospinal fluid and serum became possible only recently, with the use of sensitive and selective immunoassays (9, 11, 12).

Due to the large variation, a specific relationship of acetylcholinesterase levels to any of the diagnostic groups is not observed. Some trends are, however, apparent. For example, the values measured in bacterial and viral meningitis (groups 12 and 13) compared with the average of all values, indicate decreased levels of acetylcholinesterase in cerebrospinal fluid and serum and an increased percentage of values below the detection limit, both possibly due to enhanced degradation of proteins in both compartments. In contrast to these patients with the severe diseases, the chronic inflammatory diseases (group 4) display elevated levels of acetylcholinesterase in cerebrospinal fluid and serum and only a very few values below the detection limit. Most of the diagnostic parameters (data not shown) determined in the cerebrospinal fluid and serum of this latter group were normal. Thus groups 4 and 11 (miscellaneous) might both reflect a situation close to normal with regard to acetylcholinesterase levels, since they comprise large numbers of patients and they do not represent dramatic diseases.

The hope of finding diagnostically significant alterations has, however, not yet been fulfilled and further evaluation is necessary. The presence of a possibly 
brain-derived protein in the circulating blood, however, should encourage the search for markers of brain destruction and disease which do not rely on lumbar puncture alone.

\section{References}

1. Rasmussen, A. G., Soerensen, K., Selmer, J., Zenthen, J., Bjerrum, O. J., Brodbeck, U. \& Norgaard-Pedersen, B. (1987) Immunochemical determination of acetylcholinesterase in amniotic fluid - An evaluation of eleven monoclonal antibodies. Clin. Chim. Acta 166, 17-25.

2. Felgenhauer, K. (1988) Liquordiagnostik. In: Labor und Diagnose (Thomas, L., ed.) Med. Verlagsgesellschaft Marburg, pp. $1401-1423$.

3. Bradbury, M. (1979) The concept of a blood-brain barrier, John Wiley \& Sons, Chichester.

4. Jacobi, C., Reiber, H. O. \& Felgenhauer, K. (1986) The clinical relevance of locally produced carcinoembryonic antigen in cerebrospinal fluid. J. Neurol. 233, 358-361.

5. Chubb, J. W., Goodman, S. \& Smith, A. D. (1976) Is acetylcholinesterase secreted from central neurons into CSF? Neuroscience 1, 57-62.

6. Kreutzberg, G. W. \& Schubert, P. (1975) The cellular dynamics of intraneuronal transport. In: The use of axonal transport for studies of neuronal connectivity (Cowan, W. M. \& Cuenod, M., eds.) Elsevier, Amsterdam, pp. 85-112.

7. Soerensen, K., Brodbeck, U., Rasmussen, A. G. \& Norgaard-Pedersen, B. (1986) Determination of maternal serum acetylcholinesterase in pregnancies with neural tube defects. Prenatal Diagnosis 7, 75-79.

\section{Acknowledgement}

The actual immunochemical acctylcholincsterase measurements were performed by $K$. Soerensen in the laboratories of Prof. $U$. Brodbeck, whose encouraging support over many years is gratefully acknowledged.

8. Reiber, H. (1980) The discrimination between different blood-CSF barrier dysfunctions and inflammatory reactions of the CNS by a recent evaluation graph for the protein profile of cerebrospinal fluid. J. Neurol. 224, $89-99$.

9. Brimijoin, S. \& Hammond, P. (1988) Butyrylcholinesterase in human brain and acetylcholinesterase in human plasma: Trace enzymes measured by two-site immunoassay. J. Neurochemistry $51,1227-1231$.

10. Felgenhauer, K. (1974) Protein size and cerebrospinal fluid composition. Klin. Wochenschrift 52, 1158-1164.

11. Jacobi, C. \& Reiber, H. O. (1988) Clinical relevance of increased neuron-specific enolase concentration in cerebrospinal fluid. Clin. Chim. Acta 177, 49-54.

12. Rasmussen, A. G., Adolfsson, R. \& Karlsson, Th. (1988) New method specific acetylcholinesterase in cerebrospinal fluid: application to Alzheimer's disease. Lancet II, 571573.

Priv. Doz. Dr. M. Mäder

Neurologische Klinik der Universität Göttingen

Robert Koch Str. 40

W-3400 Göttingen 
